\title{
Short communication: A reproductive tract scoring system to manage fertility in lactating dairy cows
}

\author{
C. D. Young, ${ }^{* 1}$ F. N. Schrick, ${ }^{*}$ K. G. Pohler, ${ }^{*}$ A. M. Saxton, ${ }^{*}$ F. A. Di Croce, ${ }^{* 2}$ D. A. Roper, ${ }^{* 3}$ J. B. Wilkerson, $\dagger$ \\ and J. L. Edwards*4 \\ *Department of Animal Science, and \\ †Department of Biosystems Engineering and Soil Science, University of Tennessee, Institute of Agriculture, Knoxville 37996
}

\begin{abstract}
We developed a reproductive tract size and position score (SPS) system as a reproductive management tool to identify lactating dairy cows with decreased fertility. This system, relying solely on transrectal palpation, considers the size (cervical and uterine) and position of the reproductive tract relative to the pelvis. Cows undergoing pre-breeding exams were identified as having reproductive tracts that were small (SPS1), medium (SPS2), or large (SPS3). Cows designated SPS1 had small and compact uterine horns that rested within the pelvic cavity; SPS2 cows had reproductive tracts that were intermediate in cervical and uterine horn diameter, with longer uterine horns resting partially outside the pelvic cavity; and SPS3 cows had reproductive tracts that were larger and rested mostly outside the pelvic cavity. Cows that were SPS1 had a higher rate of pregnancy per artificial insemination $(43.3 \pm 3.7 \%)$ than cows that were SPS2 $(36.9 \pm 3.6 \%)$ or SPS3 $(27.7$ $\pm 4.3 \%)$. The percentage of cows with an SPS2 score differed in pregnancies per artificial insemination compared with SPS3 cows. The average days in milk was similar for SPS1, SPS2, and SPS3 cows (104.3 \pm 3.5 , $98.4 \pm 3.4$, and $94.7 \pm 7.7$, respectively). Ultrasound measurements of the uterine horn and cervical diameter, and length measurements of the uterine horns, cervix, and vagina confirmed differences among the SPS groups derived by transrectal palpation. The ease with which transrectal palpation can be used to determine the size and position of the reproductive tract attests to the relevance and usefulness of this scoring system to identify less fertile lactating dairy cows. The ability to do so with ease provides an opportunity to
\end{abstract}

Received November 9, 2016.

Accepted March 27, 2017.

${ }^{1}$ Current address: Zoetis, Roswell, NM 88201.

${ }^{2}$ Current address: Zoetis, Kalamazoo, MI 49007.

${ }^{3}$ Current address: Department of Animal Science and Veterinary Technology, Tarleton State University, Stephenville, TX 76402.

${ }^{4}$ Corresponding author: jedwards@utk.edu make economically relevant management decisions and maximize reproductive efficiency in a given herd.

Key words: reproductive tract size-position score, fertility, dairy cow, parity

\section{Short Communication}

Conception rates of lactating Holstein cows in the early 1980 s averaged $50.8,48.9$, and $48.3 \%$ for cows in their first, second, and third parity, respectively (Gwazdauskas et al., 1981). From 1996 to 2006, conception rates of lactating Holstein cows ranged from 33 to $30 \%$, with a low of $27 \%$ reported in 2001 (Norman et al., 2009). Although slight improvements have been noticed since 2002, attributed to increased use of estrus-ovulation synchronization protocols and better genetic selection (Norman et al., 2009; Binelli et al., 2014), additional efforts are needed to improve the reproductive performance of today's lactating dairy cows.

General evaluations of the female reproductive tract, including the diameter and tone of the uterine horns and the ovarian structures, have been used as predictors of fertility in beef (Andersen et al., 1991; Holm et al., 2009; Gutierrez et al., 2014) and dairy heifers (Stevenson et al., 2008). Relying on ultrasonography, Baez et al. (2016) reported a negative association between uterine size and fertility in lactating dairy cows. The objective of our study was to develop a reproductive tract size and position score (SPS) system that could be used as a reproductive management tool to identify lactating dairy cows with decreased fertility. To maximize implementation potential, we focused our efforts on developing a system that relied on transrectal palpation to determine the size and position of the reproductive tract relative to the pelvis. We hypothesized that pregnancy per AI would be higher in cows with smaller reproductive tracts that rested within the pelvic cavity.

We obtained institutional animal care and use approval before beginning this study. In our first study, 100 nonpregnant lactating Holstein cows (>30 DIM, corn-silage-based TMR diet) from a single herd were palpated per rectum and assigned an SPS score as de- 

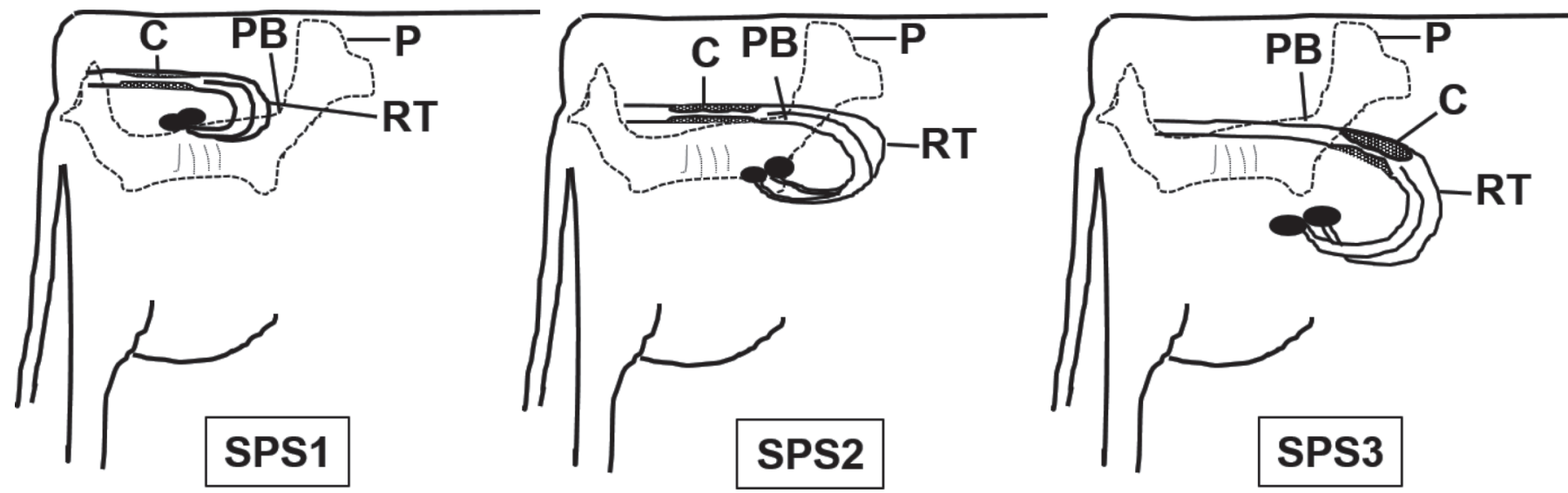

Figure 1. Depiction of reproductive tract position influence on size and position score. Reproductive tracts positioned entirely within the pelvic cavity were designated SPS1. Reproductive tracts in which the cervix is within the pelvic cavity but uterine horns are outside the pelvic cavity were designated SPS2. Reproductive tracts in which the cervix and uterine horns lie outside the pelvic cavity were designated SPS3. C $=$ cervix, $\mathrm{P}=$ pelvis, $\mathrm{RT}=$ reproductive tract, $\mathrm{PB}=$ pelvic brim, $\mathrm{SPS}=$ size and position score.

picted in Figure 1. Each cow was scored by at least 2 individuals experienced in AI who were not informed of the other's scores, and 90\% of kappa values between palpators indicated agreement $(P<0.05)$. Palpator scores were combined using a mixed model to adjust for possible differences, and the final SPS score was derived from the least squares means of that analysis. Immediately after scoring, the size of the center portion of the cervix and both uterine horns were measured using transrectal ultrasonography $(7.5 \mathrm{mHz}$ probe; MyLab Gold, Esaote, Genoa, Italy). Uterine horn diameter was obtained by measuring a cross-section approximately $2.5 \mathrm{~cm}$ beyond the external bifurcation.

We then conducted a large field trial to test the effects of SPS on fertility in lactating dairy cows ( $\mathrm{n}=$ 1,463) using 4 herds. Herd populations ranged from 175 to 700 Holstein cows housed in free stalls or bedded pack barns and fed corn-silage TMR diets. Cows were milked 3 times per day in 3 herds and twice per day in 1 herd. Cows undergoing pre-breeding reproductive exams were palpated per rectum and assigned an SPS. Cows underwent AI or timed AI $(\mathrm{n}=1,979$ inseminations) according to each herd's reproductive management plan. Inseminators were not informed of a cow's SPS before breeding. Depending on the herd, pregnancy status was determined by ultrasonography or transrectal palpation 28 to $50 \mathrm{~d}$ after insemination. Cows that were not pregnant after insemination were reassigned an SPS, and subsequent inseminations were recorded and evaluated until pregnancy was established or the cows were culled for reasons outside the scope of our study. Freshening date, insemination date, DIM at insemination, parity, service sire, location, milking frequency, milk production at insemination, and pregnancy status were recorded for each insemination. Cow were divided into 2 groups by DIM at insemination (DIMGRP) based on values above or below the mean: DIMGRP1 $\leq 116$ DIM and DIMGRP2 >116 DIM. Milk production at insemination was obtained by recording the DHIA test day or on-farm test day within $30 \mathrm{~d}$ of insemination (before or after). Cows were also divided into 2 groups by milk production (MILKGRP) based on values above or below the mean: MILKGRP1 $\leq 39$ $\mathrm{kg}$ and MILKGRP2 $>39 \mathrm{~kg}$. They were also divided into 3 parity groups: 1,2 , and 3 or more. This study was performed throughout the year, except during the summer months.

For this observational cohort study, reproductive tract measurements were analyzed using a completely randomized ANOVA. Pregnancy data were analyzed using a mixed-model ANOVA for binomial data using GLIMMIX in SAS (SAS Institute, Inc., Cary, NC). The basic model consisted of pregnancy as the dependent variable and SPS as the treatment factor. Insemination was the experimental unit. Location, service sire, DIMGRP, MILKGRP, daily milking frequency (MILKX), season or month of insemination, year of insemination, and parity were added individually, along with their interactions with SPS, as fixed effects to this base model to determine their effect on pregnancy per AI $(\mathbf{P} / \mathbf{A I})$. Milk group $(P=0.6887)$, season $(P=$ $0.2791)$, service sire $(P=0.4456)$, year $(P=0.8589)$, and parity $(P=0.0844)$ did not influence $\mathrm{P} / \mathrm{AI}$. Location $(P=0.0076)$ and MILKX $(P=0.0039)$ did have a significant effect on $\mathrm{P} / \mathrm{AI}$. Although DIMGRP did not have a significant effect as a single fixed effect $(P$ $=0.5104)$, the interaction of DIMGRP and SPS did have a significant effect $(P=0.0290)$. Location and service sire were added to the base model as random effects, and DIMGRP and MILKX were included as 
single fixed effects to make up the final model. Treatments were compared using Fisher's least significant difference mean separation $(P<0.05)$. A chi-squared test investigated an association between parity group and SPS. Repeat breeders were investigated using a chi-squared test to determine whether the frequency of repeat breeders was associated with SPS and parity. Repeat breeders were cows that had 3 or more inseminations and were not pregnant (Yusuf et al., 2010).

Initial efforts to characterize the size and position of the reproductive tract in 100 lactating dairy cows using transrectal palpation identified 36, 46, and $18 \%$ of cows with scores of SPS1, SPS2, SPS3, respectively. The use of ultrasound confirmed that cows assigned the different SPS scores did differ in the size of the uterine horns, cervix, and total diameter of structures measured (Table 1). Cows that were SPS1 or SPS2 did not differ in terms of the size of the right uterine horn, cervix, or the collective total of these structures; cows with a score of SPS3 had larger left uterine horns and higher collective total measurements of the cervix, right horn, and left horn compared with SPS1 and SPS2 cows. Cows that were SPS1 had a smaller cervical diameter than cows that were SPS3.

Vaginal length and uterine volume were also assessed in a different subset of Holstein cows assigned an SPS score before collection of their reproductive tract at an abattoir $[\mathrm{n}=35$ total; SPS1 $(\mathrm{n}=14)$, SPS2 $(\mathrm{n}=$ $11)$, SPS3 $(n=12)]$. Length of the cervix, vagina, and uterine horns was obtained using a flexible measuring tape. Cervical and vaginal measurements were taken from caudal to cranial end. Uterine horn measurements were determined from the cranial end of the cervix to the uterotubal junction of each uterine horn. The luminal volume of the uterus was estimated by placing a foley catheter into the uterine body and filling it with water until a constant pressure was achieved. Constant pressure was obtained using gravity by placing the end of the Y-tubing above the reproductive tract $(\sim 18.75$ $\mathrm{cm})$. As water entered the uterus, levels in the exit tubing rose and fell as dissipation into the reproductive tract occurred. Once the water had stabilized for 1 minute without expelling more than $2 \mathrm{~mL}$, a volume measurement was recorded. Vaginal length did not differ across SPS $(30.6,32.6$, and $32.2 \pm 1.1 \mathrm{~mm}, P=$ $0.38)$. Uterine volume averaged $97.4,115.4$, and 126.4 $\pm 19.7 \mathrm{~mL}$ across SPS, respectively. Baez et al. (2016) have reported that uterine volume differs across cows separated into quartiles by uterine size.

We then conducted a field trial to determine the extent to which SPS scores of the reproductive tract relate to $\mathrm{P} / \mathrm{AI}$ success in lactating dairy cows. A total of 1,463 cows were enrolled in the study and assigned an SPS. Within a single parity, SPS changed in only $3.1 \%$ of the cows scored $(\mathrm{n}=46 / 1463)$. Cows initially scored as SPS2 had the highest percentage of change within a single parity $(5.5 \%, 32 / 586)$, compared with SPS1 cows $(1.6 \%, 12 / 747)$ and SPS3 cows $(1.5 \%, 2 / 130)$. The frequency of SPS1 cows decreased as parity increased, and the frequency of SPS2 and SPS3 cows increased as parity increased (Figure 2A). Independent of parity, average DIM were similar in SPS1, SPS2, and SPS3 cows $(104.3 \pm 3.5,98.4 \pm 3.4$ and $94.7 \pm 7.7$ DIM, respectively).

Cows assigned a score of SPS1 using transrectal palpation had a higher $\mathrm{P} / \mathrm{AI}$ than cows assigned a score of SPS2 $(P=0.006)$ or SPS3 $(P=0.0002$; Figure $2 \mathrm{~B})$. Furthermore, SPS2 cows had a higher P/AI than SPS3 cows $(P=0.03)$. Overall, $18.3 \%(190 / 1039)$ of cows that underwent AI were identified as repeat breeders. Within this category, the percentage of SPS1 cows was lower $(14.5 \%, \mathrm{n}=76 / 525)$ than the percentage of SPS2 cows $(21.5 \%, \mathrm{n}=92 / 428)$ or SPS3 cows $(25.6 \%, \mathrm{n}=$ $22 / 86 ; P=0.0039)$. However, SPS1 cows had a longer interval to reinsemination ( $28 \pm 0.5 \mathrm{~d})$ than SPS2 cows $(25.4 \pm 0.5 \mathrm{~d} ; P<0.0001)$ and SPS3 cows $(25.7 \pm 0.9$ $\mathrm{d} ; P=0.02)$.

Our overarching goal was to develop a reproductive tract scoring system for lactating dairy cows to improve $\mathrm{P} / \mathrm{AI}$ success. A system such as this would provide the

Table 1. Ultrasound measurements (mm; \pm SEM) for different size and position scores (SPS) ${ }^{1}$

\begin{tabular}{lcccc}
\hline Structure & $\begin{array}{c}\text { SPS1 } \\
(\mathrm{n}=36)\end{array}$ & $\begin{array}{c}\text { SPS2 } \\
(\mathrm{n}=46)\end{array}$ & $\begin{array}{c}\text { SPS3 } \\
(\mathrm{n}=18)\end{array}$ & $P$-value \\
\hline Left horn & $14.0 \pm 0.6^{\mathrm{a}}$ & $15.5 \pm 0.5^{\mathrm{b}}$ & $17.5 \pm 0.8^{\mathrm{c}}$ & 0.0020 \\
Right horn & $15.4 \pm 0.6^{\mathrm{a}}$ & $15.2 \pm 0.5^{\mathrm{a}}$ & $17.1 \pm 1.0^{\mathrm{a}}$ & 0.1928 \\
Cervix $_{\text {Total }^{2}}$ & $21.3 \pm 0.9^{\mathrm{a}}$ & $23.5 \pm 0.8^{\mathrm{ab}}$ & $26.0 \pm 1.3^{\mathrm{b}}$ & 0.0135 \\
\hline
\end{tabular}

$\overline{\mathrm{a}, \mathrm{b}}$ Means with a different superscript letter within a row differ $(P<0.05)$.

${ }^{1}$ Cows designated SPS1 had small and compact uterine horns that rested within the pelvic cavity; SPS2 cows had reproductive tracts that were intermediate in cervical and uterine horn diameter, with longer uterine horns resting partially outside the pelvic cavity; and SPS3 cows had reproductive tracts that were larger and rested mostly outside the pelvic cavity.

${ }^{2}$ Sum of diameter measurements of left horn, right horn, and cervix. 

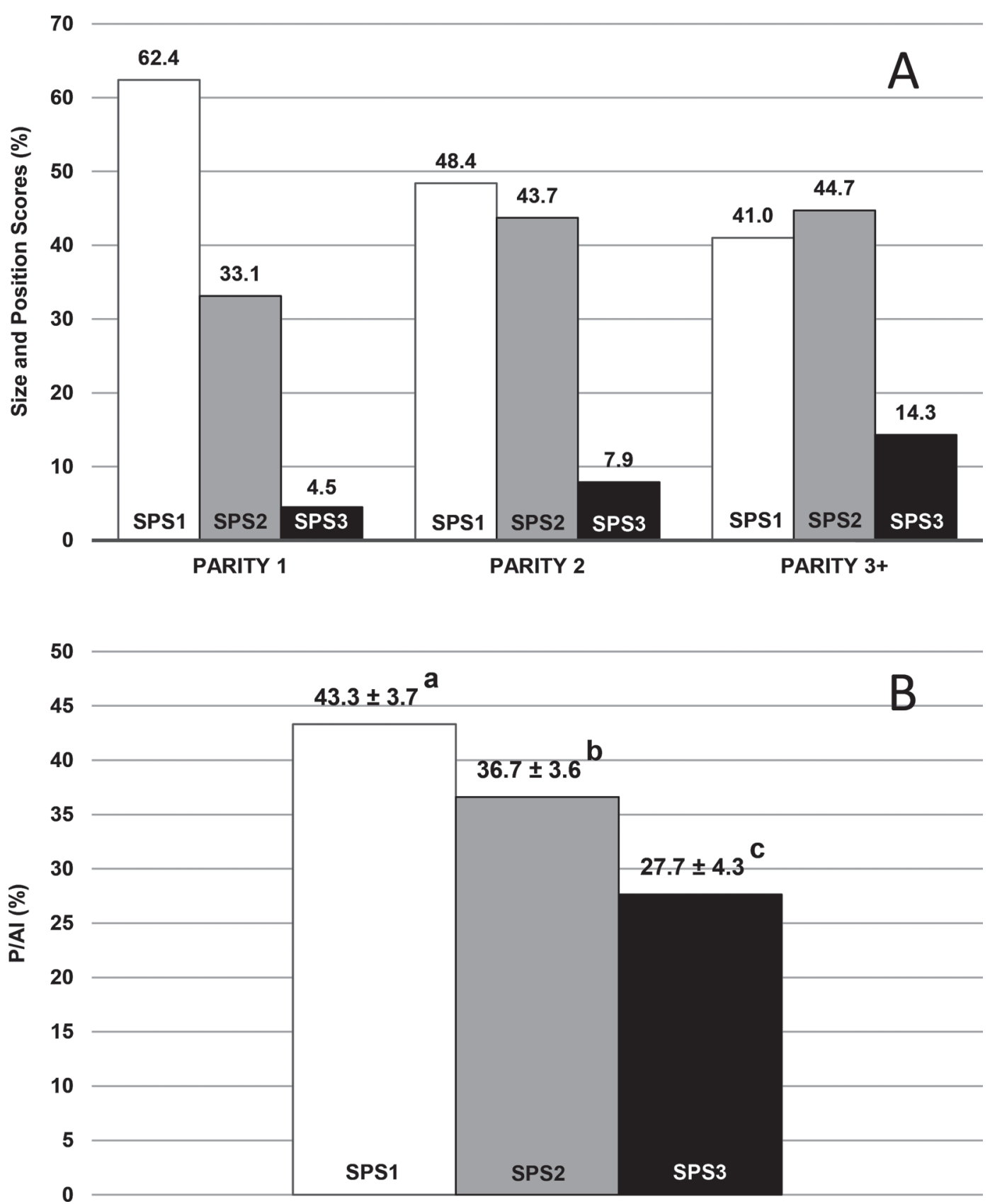

Figure 2. (A) Influence of parity on size and position score (SPS). Cows designated SPS1 had small and compact uterine horns that rested within the pelvic cavity; SPS2 cows had reproductive tracts that were intermediate in cervical and uterine horn diameter, with longer uterine horns resting partially outside the pelvic cavity; and SPS3 cows had reproductive tracts that were larger and rested mostly outside the pelvic cavity. Percentages of SPS1 decreased as parity increased over parity 1,2 , and 3 or more $(\mathrm{n}=387 / 620,153 / 316$, and $235 / 573$, respectively). Percentages of SPS2 increased as parity increased over parity 1,2 , and 3 or more $(\mathrm{n}=205 / 620,138 / 316$, and $256 / 573$, respectively). Percentages of SPS3 increased as parity increased over parity 1,2 , and 3 or more $(\mathrm{n}=28 / 620,25 / 316$, and $82 / 573$, respectively; chi-squared $P<0.0001$ ). (B) Pregnancy per artificial insemination (P/AI) of size and position scores. The P/AI for cows with SPS1 (43.3 $\pm 3.7 \%, \mathrm{n}=929)$ were greater than for SPS2 $(36.7 \pm 3.6 \%, \mathrm{n}=871 ; P=0.0055)$ and SPS3 $(27.7 \pm 4.3 \%, \mathrm{n}=179 ; P=0.0002)$. The $\mathrm{P} / \mathrm{AI}$ for cows with SPS2 was also greater than for cows with SPS3 $(P=0.0268)$. Different letters $(\mathrm{a}-\mathrm{c})$ represent a difference $(P<0.05)$ among the means.

dairy producer with an additional opportunity to make economically important management-based decisions. We prioritized the development of a system that relied on palpation per rectum to derive scores where obvious differences existed related to the size and position of the reproductive tract, to ensure the relevance and potential for adoption by individuals proficient in AI (e.g., dairy producers, AI technicians, and veterinarians). Develop- 
ing an approach based on size and position is especially appealing, because individuals proficient in AI expect the size and position of the reproductive tract to vary in cows but may not be using this information to make important management decisions.

In the present study, most cows in the 4 different herds presented with a small- to moderate-sized reproductive tract; all or much of it was contained within the pelvic cavity. These cows were given a score of SPS1 or SPS2 (Figure 1) and represented $\sim 80$ to $90 \%$ of cows palpated per rectum. A subset of cows with larger reproductive tracts positioned outside the pelvic cavity were scored as SPS3, made up $\sim 10$ to $20 \%$ of animals palpated per rectum, and were found in each of the parity groups $(1$, 2 , and $3+$ ). Ultrasound confirmed that scoring criteria were effective for categorizing reproductive tract sizes into 3 different groups with respect to the size of the uterine horns and cervix, and the total diameter of the structures measured. Based on data obtained from a smaller subset of cows scored at an abattoir before reproductive tracts were collected, vaginal length was similar in SPS1-3 cows. Although the position of the reproductive tract in situ may be influenced by size and weight, the dimensions of the pelvic floor and cavity, along with possible differences in the integrity of the broad ligament, are likely contributing factors.

Nonetheless, the ease with which the relative size and position of the reproductive tract can be determined by palpation per rectum and then categorized, highlights the potential usefulness of our scoring system. Relevant for identifying less fertile cows, $\mathrm{P} / \mathrm{AI}$ was $\sim 15$ percentage points less in cows with larger reproductive tracts positioned outside the pelvic cavity (SPS3) than in those with smaller reproductive tracts positioned in the pelvic cavity (SPS1). Stark differences in P/AI percentage points for SPS1 versus SPS3 cows likely related to differences in reproductive tract size. In a recent study by Baez et al. (2016), more of the cows that became pregnant after synchronization with a Double Ovsynch protocol had a smaller uterine diameter (ultrasound) and volume (estimated using uterine measurements) 72 $\mathrm{h}$ before timed AI. We also observed a tendency for pregnant cows to have a reduced uterine length. Baez et al. (2016) also reported a difference of 12 percentage points in $\mathrm{P} / \mathrm{AI}$ of in cows with a uterine volume of $\leq 128 \mathrm{~cm}^{3}$ versus $>128 \mathrm{~cm}^{3}$, attesting to the influences of reproductive tract size on fertility. Uterine volumes were calculated differently in their study, limiting direct comparison with the values from the present study.

As parity increased, the frequency of cows in our study with smaller reproductive tracts located in the pelvic cavity (i.e., SPS1) decreased (i.e., reproductive tract scores of SPS2 and SPS3 increased as parity increased; Figure 2A). This finding was consistent with Baez et al. (2016), who reported increases in uterine size with increasing parity. Whether changes in size-position are sufficient to explain some of the reported differences in fertility in multiparous versus primiparous cows is unclear (Stevenson and Phatak, 2010; Herlihy et al., 2012; Pursley et al., 2012). However, in the present study, multiparous SPS1 cows (parity: $3+$ ) had P/AI (40.6 \pm $2.8 \%)$ similar to parity $1(41.7 \pm 2.5)$ and parity $2(41.3$ $\pm 3.3 \%)$ SPS1 cows. This finding emphasizes that reproductive tract size-position is likely more influential on fertility than parity alone.

Cows were identified as repeat breeders in our study when they did not become pregnant after 3 inseminations. We obtained an $18.3 \%$ overall occurrence of repeat breeders, consistent with the 14 to $24 \%$ reported by others (Bartlett et al., 1986; Yusuf et al., 2010). The percentage of repeat breeder cows increased as parity increased $(P=0.0039)$. Relevant for reproductive tract scoring, the percentage of SPS1, SPS2, and SPS3 repeat breeders were $14.5,21.5$, and $25.6 \%$, respectively. The frequency of repeat breeder cows in the SPS3 group was 11 percentage points higher than in the SPS1 group, suggesting that reproductive tract size-position is problematic in this subset of cows.

Although rectal palpation alone is not an accurate predictor of fertility, efforts to do so while being mindful of the size and position of the reproductive tract are likely to be informative, especially when accounting for cervical size. Larger cervices at 27 to 33 DIM have been related to poor fertility (LeBlanc et al., 2002). In our study, SPS3 cows had larger cervices than SPS1 cows. Whether larger cervices in certain subsets of cows relate to a previous or existing disorder is unclear, although cervical inflammation has been related to decreased pregnancy outcomes. Deguillaume et al. (2012) reported that endometrial inflammation existed in $\sim 75 \%$ of cows with cervical inflammation. In our study, cows with an obvious reproductive disorder at the time of SPS assignment were not scored until after it had resolved. Because information related to a previous puerperium disorder was not available before scoring, factors contributing to a larger cervix, uterus, or both in SPS3 cows are not clear.

The present study documents the usefulness of a scoring system based on the size and position of the reproductive tract to identify a less fertile subset of cows, especially those with a large reproductive tract positioned outside the pelvic cavity. Our finding that cows with a large reproductive tract had a $\mathrm{P} / \mathrm{AI} \sim 15$ percentage points less than cows with a smaller reproductive tract positioned in the pelvic cavity highlights just how costly it is to breed this subset of cows. To breed SPS3 cows or not is an important question for economics-based management decisions. When repro- 
ductive culling is not desirable, the use of semen that is less expensive or that comes from bulls with a high conception rate may be warranted. Related to the use of sexed semen, efforts to prioritize the insemination of SPS1 cows may be important for maximizing fertility. The ability to obtain similar P/AI for SPS1 cows regardless of parity-age also provides information that may be useful for determining the retention of certain cows in a given herd. Depending on the extent to which the size and position of the reproductive tract relates to a nonobvious postpartum disorder, this scoring system may also be useful for early identification and treatment.

\section{ACKNOWLEDGMENTS}

This research was supported in part by the state of Tennessee through UT AgResearch and the Department of Animal Science. We thank The University of Tennessee AgResearch and Education Centers and their personnel, commercial dairies (Harrison Dairy, Sweetwater Valley Farms and Pate Acres Dairy), and practitioners for their participation in this study. The authors also thank Felipe Dantas and Rebecca Payton (University of Tennessee, Knoxville) for critical review of this manuscript.

\section{REFERENCES}

Andersen, K. J., D. G. Lefever, J. S. Brinks, and K. G. Odde. 1991. The use of reproductive tract scoring in beef heifers. Agric. Practice $12: 19-26$.

Baez, G. M., R. V. Barletta, J. N. Guenther, J. M. Gaska, and M. C. Wiltbank. 2016. Effect of uterine size on fertility of lactating dairy cows. Theriogenology 85:1357-1366.

Bartlett, P. C., J. H. Kirk, and E. C. Mather. 1986. Repeated insemination in Michigan Holstein-Friesian cattle: Incidence, descriptive epidemiology and estimated economic impact. Theriogenology 26:309-322.

Binelli, M., R. Sartori, J. L. M. Vasconcelos, P. L. J. Monteiro, M. H. C. Pereira, and R. S. Ramos. 2014. Evolution in fixed-time: From synchronization of ovulation to improved fertility. Pages 493-506 in Reproduction of Domestic Ruminants VIII. Context Products Ltd., Leicestershire, UK.

Deguillaume, L., A. Geffré, L. Desquilbet, A. Dizien, S. Thoumire, C. Vornière, F. Constant, R. Fournier, and S. Chastant-Maillard. 2012. Effect of endocervical inflammation on days to conception in dairy cows. J. Dairy Sci. 95:1776-1783.

Gutierrez, K., R. Kasimanickam, A. Tibary, J. M. Gay, J. P. Kastelic, J. B. Hall, and W. D. Whittier. 2014. Effect of reproductive tract scoring on reproductive efficiency in beef heifers bred by timed insemination and natural service versus only natural service. Theriogenology 81:918-924.

Gwazdauskas, F. C., J. A. Lineweaver, and W. E. Vinson. 1981. Rates of conception by artificial insemination of dairy cattle. J. Dairy Sci. 64:358-362.

Herlihy, M. M., J. O. Giordano, A. H. Souza, H. Ayres, R. M. Ferreira, A. Keskin, A. B. Nascimento, J. N. Guenther, J. M. Gaska, S. J. Kacuba, M. A. Crowe, S. T. Butler, and M. C. Wiltbank. 2012. Presynchronization with Double-Ovsynch improves fertility at first postpartum artificial insemination in lactating dairy cows. J. Dairy Sci. 95:7003-7014.

Holm, D. E., P. N. Thompson, and P. C. Irons. 2009. The value of reproductive tract scoring as a predictor of fertility and production outcomes in beef heifers. J. Anim. Sci. 87:1934-1940.

LeBlanc, S. J., T. F. Duffield, K. E. Leslie, K. G. Bateman, G. P. Keefe, J. S. Walton, and W. H. Johnson. 2002. Defining and diagnosing postpartum clinical endometritis and its impact on reproductive performance in dairy cows. J. Dairy Sci. 85:2223-2236.

Norman, H. D., J. R. Wright, S. M. Hubbard, R. H. Miller, and J. L. Hutchison. 2009. Reproductive status of Holstein and Jersey cows in the United States. J. Dairy Sci. 92:3517-3528.

Pursley, J. R., J. P. N. Martins, C. Wright, and N. D. Stewart. 2012. Compared to dinoprost tromethamine, cloprostenol sodium increased rates of estrus detection, conception and pregnancy in lactating dairy cows on a large commercial dairy. Theriogenology $78: 823-829$

Stevenson, J. L., J. A. Rodrigues, F. A. Braga, S. Bitente, J. C. Dalton, J. E. P. Santos, and R. C. Chebel. 2008. Effect of breeding protocols and reproductive tract score on reproductive performance of dairy heifers and economic outcome of breeding programs. J. Dairy Sci. 91:3424-3438.

Stevenson, J. S., and A. P. Phatak. 2010. Rates of luteolysis and pregnancy in dairy cows after treatment with cloprostenol or dinoprost. Theriogenology 73:1127-1138.

Yusuf, M., T. Nakao, R. M. S. B. K. Ranasinghe, G. Gautam, S. T. Long, C. Yoshida, K. Koike, and A. Hayashi. 2010. Reproductive performance of repeat breeders in dairy herds. Theriogenology $73: 1220-1229$. 